\title{
Performance Analysis of SPE-OCDMA over WDM Networks
}

\author{
K. Puntsri *, S. Sittichivapak ${ }^{* *}$ and H.M.H.Shalaby ${ }^{* * *}$ \\ * Rajamangala University of Technology Isan (Khon Kean Campus), Department of Electronic and \\ Telecommunication Engineering, Khon Kean, Thailand \\ ** King Mongkut's Institute of Technology Ladkrabang, Department of Telecommunications Engineering, Bangkok, Thailand \\ ${ }^{* * *}$ University of Alexandria, Department of Electronic Engineering, Alexandria, Egypt \\ e-mail kidsanapong.pu@rmuti.ac.th, kssuvepo@kmitl.ac.th, shalaby@ieee.org
}

\begin{abstract}
This paper presents a performance analysis of SPEOCDMA (spectrally phase-encoded optical CDMA) over WDM networks. The discrete time Markov chain model is used to build analytical model of multi-channel random access Slotted Aloha. The number of WDM wavelength is corresponded to multichannel Slotted Aloha. We measure several performance characteristics, namely, steady-state throughput and average packet delay time. Additionally, the efficiencies of the system, which are based on both the processing gain and the number of WDM wavelengths, are considered. Additionally, exponential retransmission is used for solving packet collision problems. In our numerical results, system performance is given in terms of both average throughput and delay time.
\end{abstract}

\section{INTRODUCTION}

Nowadays, OCDMA (Optical code division multiple access), especially, spectrally phase-encoded optical CDMA has been widely developed as high-rate optical communication network systems, where encoding and decoding are all performed in optical domain [1-2,6,8]. The advantageous function of the optical CDMA is the fact that it lets each user access the network asynchronously and simultaneously without strict wavelength control and timing synchronization. In addition, optical CDMA local area networks allow shared access to a broadcast medium. However, multiple access interference (MAI) in OCDMA increases as the number of users increases.

In this paper, we propose a hybrid wavelength division multiplexing/spectrally phase-encoded optical CDMA (SPEOCDMA) over WDM network. SPE-OCDMA or coherent ultra-short light pulse techniques seems to be a good technique, since it offers high bit rates [6,8]. SPE-OCDMA technique is used to encode/decode data with the OCDMA code sets. Each simultaneous user or node is assigned a different unique code. The encoded data is then sent to wavelength division multiplexing (WDM) channels. Multi-channel slotted Aloha is used to contend free WDM wavelengths for each OCDMA code (each node contends the available wavelengths for sending packets out). We assume that the number of channels equal to the number of wavelengths. Packet collision occurs if more than two active users choose the same wavelength and same CDMA code at the same time. Therefore, to resolve this problem, exponential retransmission is used. We measure our system performance using both average system steady-state throughput and average system packet delay time. Both are based on discrete time Markov chain model.

The rest of this paper is organized as follows. In section 2, the system model description is presented. The mathematical analysis is illustrated in section 3 , where we give more details about discrete time Markov chain. In section 4, the numerical results are shown in terms of both system throughput and delay time versus the number of active users. Finally the conclusions are presented in section 5 .

\section{PeRformance ANALysis}

\section{A. System consideration}

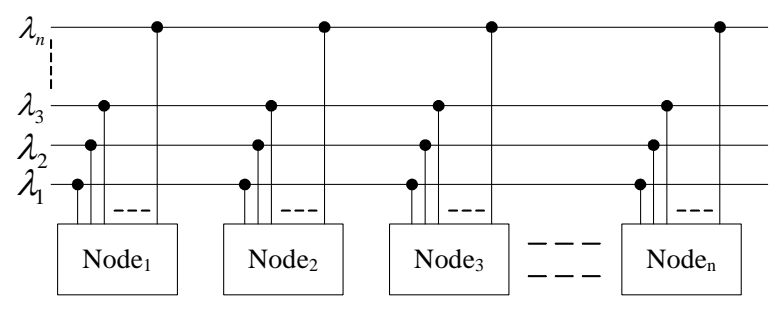

Figure 1. Architecture of optical CDMA code over WDM channel

From the system mode, the node composes of a set of transmitters (data sources and tunable lasers, optical CDMA encoders), and a set of receivers (optical CDMA decoders). The data is encoded/decoded with the set of spectrally phaseencoded optical CDMA (SPE-CDMA) technique [6,8]. Each encoded data is assigned a WDM wavelength by using multichannel random access MAC protocol. That is, on each WDM wavelength, $N$ users can be accommodated by individually assigning each user with a different optical CDMA code. Therefore, the same code sequence can be reused on all WDM channels. We use Multi-channel slotted Aloha random access protocol to assign WDM wavelengths $\lambda_{i}, i=1,2,3, \ldots n$ [5-6], as shown in Fig. 1.

We assume that the packet is received successfully if no collision has occurred (no terminals or nodes have chosen the same wavelength and same code at the same time to transmits 
packets out). In reality, this problem is unavoidable, therefore, in this paper, the exponential backoff retransmission with probability of retransmission $p=r^{n}$ is used to solve this problem, $n=0,1,2, \ldots, k$ is level of retransmission.

\section{B. Probability of Packet errors for SPE-OCDMA}

We assume that if the data bit to be transmitted is " 0 ", then no spectral-phase encoder energy is transmitted. On the other hand, if the data bit is " 1 ", an ultrashort pulse is sent out to the spectral-phase encoder. The receiver declares a " 1 " was sent if the decoded intensity exceed the threshold and declares a " 0 " otherwise. Therefore, the probability of bit error $P_{e}$ for SPE optical CDMA is given by [8],

$$
\begin{aligned}
P_{e}= & \left(\frac{1}{2}\right) \cdot\left[\sum_{l=1}^{N-1}\left(\begin{array}{c}
N-l \\
l
\end{array}\right)\left(\frac{1}{2 K}\right)^{l}\left(1-\frac{1}{2 K}\right)^{N-1-l}\right. \\
& \cdot(1-(\gamma(l)-\rho(l)))]
\end{aligned}
$$

Where $K=T_{b} / T$ is the processing gain, $T_{b}$ is period of the data source, $T$ is the encoded pulse duration,

$$
\gamma(l)=1-\exp \left(-I_{t h} N_{0} / l P_{0}\right)
$$

and

$$
\rho(l)=1-Q\left(\sqrt{\frac{2 N_{0}}{l}}, \sqrt{\frac{2 N_{0} I_{\text {th }}}{l P_{0}}}\right)
$$

Here $Q(a, b)$ is the Marcum's $Q$ function, expressed as:

$$
Q(a, b)=\int_{b}^{\infty} x \cdot \exp \left(-\frac{a^{2}+b^{2}}{2}\right) I_{0}(a x) d x
$$

$P_{0}$ is the peak power of the ultrashort pulse, $N_{0}$ is the number of chips in the coded sequence, and $I_{\text {th }}$ is the intensity threshold of the receiver comparator for detecting 0 and 1 . Therefore, from the probability of packet error in (1), the success probability of $L$ bits length can be given in (5),

$$
\begin{aligned}
P_{s}(n)= & \left(1-\frac{1}{2 K}\right)^{N-1} \\
& +\left[\sum_{l=1}^{N-1}\left(\begin{array}{c}
N-1 \\
l
\end{array}\right)\left(\frac{1}{2 K}\right)^{l}\left(1-\frac{1}{2 K}\right)^{N-1-l}\right. \\
& \left.\cdot\left(1-\frac{1}{2}(1-(\gamma(l)-\rho(l)))^{L}\right)\right]
\end{aligned}
$$

\section{System Performance Analysis}

In this section, we analyze the performance of our system using discrete Markov chain model [10]. We assume that the number of channels equal to the number of wavelengths. Packet collision occurs if more than one active user chooses the same wavelength and the same code at the same time. Therefore, to resolve this problem, the exponential retransmission is used. However, if more that one active user select only same wavelength (but different codes) at the same time, there will be multiple access interference (MAI), no collision.

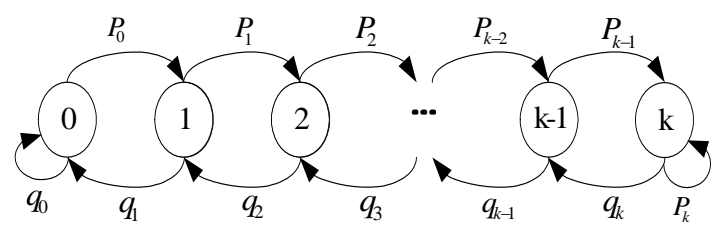

Figure 2. State transitions of Markov chain model of exponential retransmission level

The discrete time Markov chain is shown in Fig. 2. Let $p(t)$ be the retransmission probability in the $\mathrm{t}$-th slot (equal period of a packet length). $\quad p(t)$ is updated according to acknowledgment feedback. There are idle and busy statuses, in the busy statuses correspond to both success and collision of the current time slot, it could be expressed as $p(t+1)=\min \left(p_{\max }, p(t) / r\right)$, if number of idle wavelengths $\geq\lfloor x \cdot M\rfloor$ and $p(t+1)=\max \left(p_{\min }, p(t) \cdot r\right)$, if number of wavelengths $<\lfloor x \cdot M\rfloor$

Where $\lfloor x\rfloor$ denotes the largest integer not greater than $x$. $0<x<1$ be the factor of wavelengths remain in system, $0<r<1$ be the probability of retransmission when collision occurred, $0<p_{\max } \leq 1$, and $p_{\min }=r^{k}$ and $k$ be the retransmission level.

Noted that, increasing the $k$ level guarantees system stability but with large system delay time.

The system state transition probability from state $n$ to $j$,

$P_{(n, j)}$ is present in (6) [10]

$$
P_{(n, j)}= \begin{cases}q_{0} & , n=j=0 \\ p_{k} & , n=j=k \\ p_{n} & , j=n+1 \text { and } 0 \leq n \leq k-1 \\ q_{n} & , j=n-1 \text { and } 0<n \leq k\end{cases}
$$

$$
\text { when } n=0,1,2, \ldots k
$$

Here, by applying binomial distribution, we get the probability that number of idle WDM wavelengths $<\lfloor M \cdot x\rfloor p_{n}$ when system state $n$ is 


$$
p_{n}=\sum_{j=0}^{\lfloor M \cdot x\rfloor-1}\left(\begin{array}{c}
M \\
j
\end{array}\right)\left\{\left(1-\frac{r^{n}}{M}\right)^{N}\right\}^{j} \cdot\left\{1-\left(1-\frac{r^{n}}{M}\right)^{N}\right\}^{M-j}
$$

Hence, $N$, is the number of stations or nodes

$M$, is the number of WDM wavelengths and $q_{n}$ is the probability that number of idle wavelengths is $>\lfloor M \cdot x\rfloor$

$$
q_{n}=1-p_{n}
$$

Let $\pi$ and $P$ be the limiting probability vector and the transition probability matrix, respectively. Therefore, the limiting probability can be satisfied the steady-state probability from,

$$
\pi=\pi P \text {, and } \sum_{i=0}^{k} \pi_{i}=1
$$

the overall system throughput $S_{o a}(N)$ given by,

$$
\begin{aligned}
& S_{\text {oa }}(N)=\sum_{j=0}^{N}\left\{\left(\begin{array}{c}
N \\
j
\end{array}\right)\left(r^{n}\right)^{j}\left(1-r^{n}\right)^{N-j}\right. \\
& \left.\cdot j\left(1-\frac{1}{M}\right)^{j-1}\right\} \cdot P_{S}(N)
\end{aligned}
$$

And throughput per wavelength $S_{p c}(N)$,

$$
S_{p c}(N)=\frac{1}{M} \cdot S_{o a}(N)
$$

the steady-state system throughput with spectrally phaseencoded optical CDMA is given in $(12)[3-5,10]$ and $P_{s}$ was given in A of Section II,

$$
S=\sum_{n=0}^{N} \pi_{n} S(N)
$$

Here,

$$
s(N)=\left\{\begin{array}{l}
S_{p c}(N), \\
\text { for throughput per wavelength } \\
S_{o a}(N), \\
\text { for overall throughput }
\end{array}\right.
$$

Finally the system packet delay is,

$$
D=1+\frac{1}{S} \sum_{n=0}^{N} n \pi_{n}
$$

\section{NUMERICAL RESULTS}

In this section, we present some numerical results in terms of system throughput and system delay time for the spectrally phase-encoded optical CDMA over WDM networks via random access protocol. In all of our calculations of system performance, we select same parameters as given in [3-5,8], namely, packet length $L=128$ bits, $N_{0}=32, I_{\text {th }} / P_{o} \approx 1 / 4$, $x=0.5$.

In Fig. 3 and 4, we compare the system throughput per wavelength and system packet delay time per wavelength while varying the processing gain $K$ from 2 to 4 with $r=0.9$ (considered high traffic load on network)., respectively, and holding the wavelength fixed at 22 wavelengths.

From Fig. 3 we see that, the throughput of processing gain with $K=4$ is more than that of $K=2$ by 2.5 times. In this case, the maximum throughput is about 2.8, but for $K=2$ the maximum throughput is about 1.2.

Fig. 4 shows that the system packets delay time with $K=4$ is less than $K=2$, corresponding to system throughput in Fig. 3. Therefore, we see that, when the processing gain is increased the system delay time decreases, and the system throughput increases as well.

In Fig. 5 and 6, we compare the over all system throughput and system packet delay time while varying the number of wavelengths at 18, 20 and 22 wavelengths by using (10). We again observe both the maximum throughput in Fig. 5 and minimum delay time in Fig. 6, respectively. We saw that, the over all system performance increased when the number of wavelengths increased. It is clear that, the processing $(K)$ and the number of wavelength strongly affect the system performance when the SPE-OCDMA is used.

In Fig. 7 and Fig. 8, we plot the system throughput and system packet delay time per wavelength while varying the probability of retransmission $r$. It could be seen that when the number of $r$ increased, the system throughput decreases but system delay time increases cause traffic on network is increased

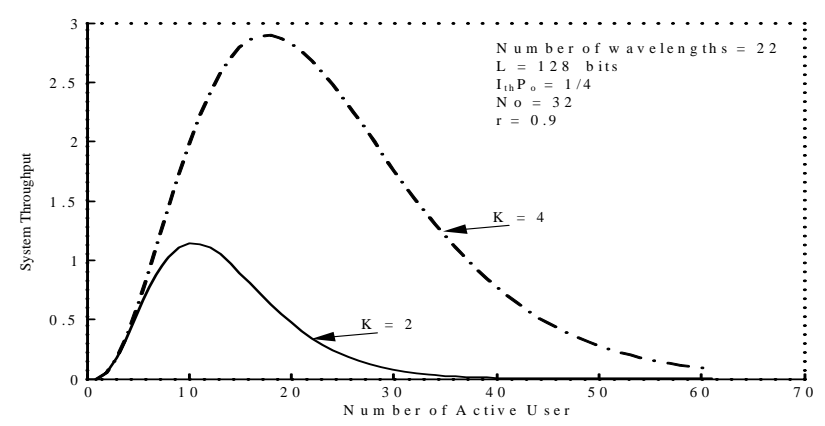

Figure 3. System throughput per wavelength versus number of active user with various $K$ (Processing gain) 


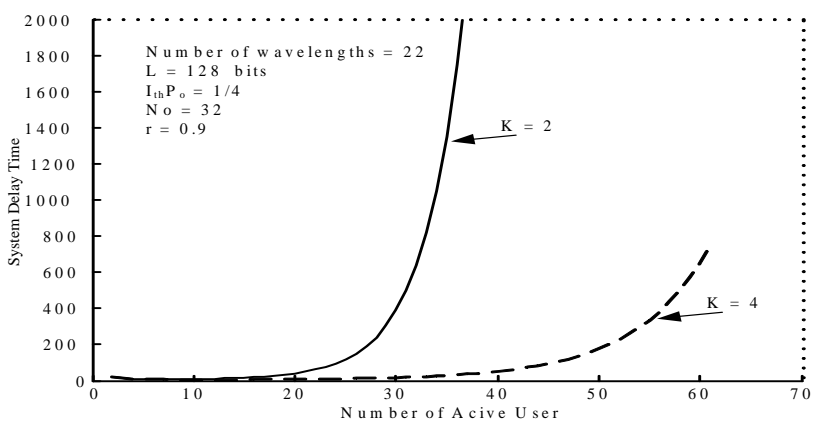

Figure 4. System packet delay time versus number of active user with various $K$ (Processing gain)

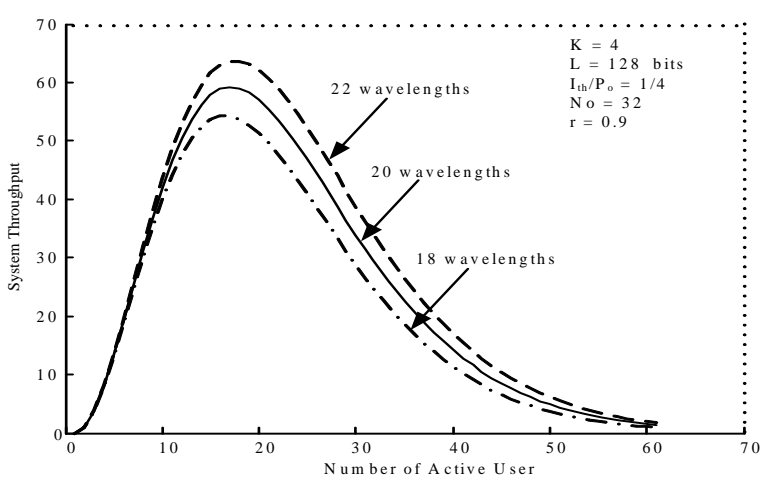

Figure 5. System throughput versus number of active users with various WDM wavelengths

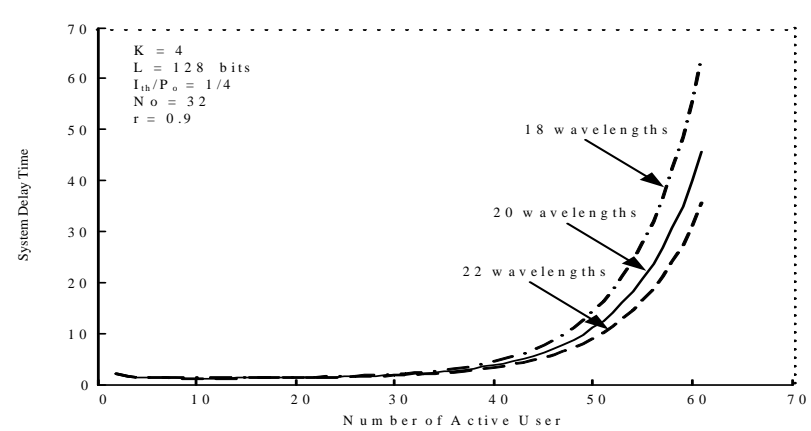

Figure 6. System packet delay time versus number of active users with various WDM wavelengths

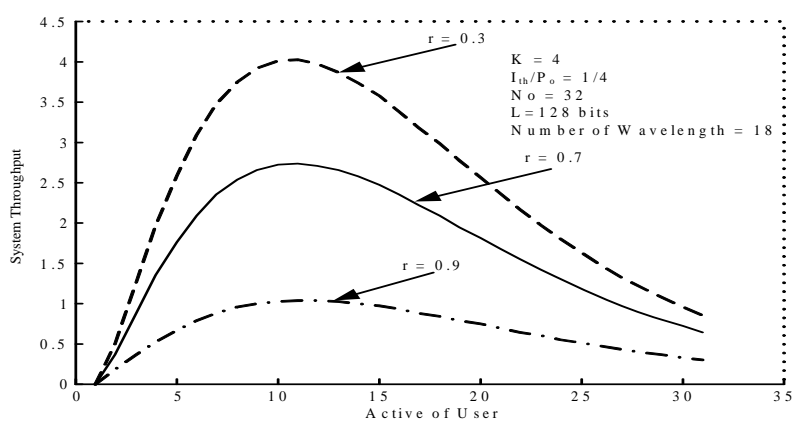

Figure 7. System throughput versus number of active users with various probability of retransmission

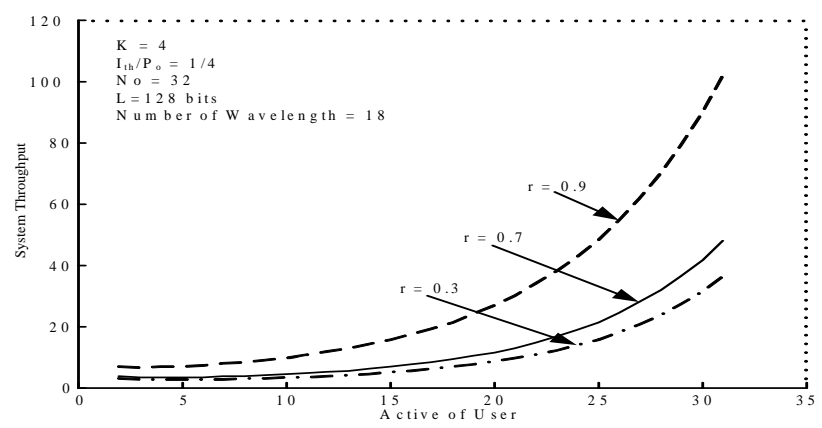

Figure 8. System packet delay time versus number of active users with various probability of retransmission

\section{CONCLUSIONS}

We propose a performance analysis of SPE-OCDMA over WDM networks via random access protocol. The SPEOCDMA technique is used to encode/decode data with the OCDMA code sets. Each simultaneous user or node is assigned a different unique code. Additionally, multi-channel Slotted Aloha is used to contend the available WDM wavelengths. The two characteristics of system performance are considered. One is system steady-state throughput and another is average system packet delay time. The results show that, the processing gain, and high traffic load (probability of retransmission increased), and the number of wavelengths have more effects in system. Therefore, when we use SE-OCDMA technique for optical fiber communication, these factors and effects should be considered.

\section{REFERENCES}

[1] Chao-Chin Yang. "Hybrid wavelength-Division-Multiplexing/SpectralAmplitude-Coding Optical CDMA System,” IEEE Photonics Technology Letters, 2005, vol. 17.

[2] Kitayama, K, Xu Wang, Naoya Wada. "OCDMA over WDM PONsolution path to gigabit-symmetric FTTH," Journal of Lightwave Technology, 2006, vol. 24, Issue 4:1654 - 1662.

[3] H.M.H. Shalaby. "Optical CDMA random access protocols with and without pretransmission coordination," IEEE J. Lightwave Technol., 2003, vol. 21: 2455-2462.

[4] H.M.H. Shalaby. "Performance Analysis of an Optical CDMA Random Protocol,” IEEE J. Lightwave Technol., 2004, vol. 22, No. 5:1233-1241.

[5] Stok, A., Sargent, E.H. "System performance comparison of optical CDMA and WDMA in a broadcast local area network," IEEE Communications Letters, 2004, vol. 6, Issue 9: 409 - 411.

[6] Galli, S., Menendez, R., Toliver, P., Banwell, T., Jackel, J., Young, J., Etemad, S. "DWDM-compatible spectrally phase encoded optical CDMA,” GLOBECOM '04. IEEE, 2004, Vol.3:1888 - 1894.

[7] Kamath, P., Touch, J.D., Bannister, J.A. "The need for media access control in optical CDMA networks,” INFOCOM 2004, vol. 4, March 711, 2004: 2208 - 2219,

[8] K. Sina, K. Salman, and J. Kambiz. "Analysis of Throughput and Delay in a Spectrally Phase-Encoded Optical CDMA Packet Network," the WOCN '07 Conference, 2007, 2-4 July 2007.

[9] D. Benhaddou, A. Al-Fuqaha, and G. Chaudhry. "New Multiprotocol WDM/CDMA-based Optical Switch Architecture," the 34th Proceedings of Simulation Symposium 2001, April: 285-291.

[10] M.S. Alam, A.Z.M.E. Hossain. "Throughput Analysis of a Multi-channel Slotted-ALOHA Protocol in Short-haul Communication Environment for an Exponential Backoff Retransmission Scheme," The Proceedings of ICICS 1997, vol.2, Sept. 9-12, 1997: 1034 - 1038. 